\title{
A new weight compensation model considering joint misalignments monitored by a feedforward impedance control method applied to an active upper-limb exoskeleton
}

Dorian Verdel ${ }^{1,2 *}$, Simon Bastide ${ }^{1,2 \dagger}$, Nicolas Vignais ${ }^{1,2}$, Olivier Bruneau ${ }^{3}$ and Bastien Berret ${ }^{1,2,4}$

\begin{abstract}
Background: Active exoskeletons are promising devices for improving rehabilitation procedures in patients. In particular, exoskeletons implementing human limb's weight support (WS) are interesting to restore some mobility in patients with muscle weakness. Using active exoskeletons should result in accurate and generic WS but its effect on human motor control will critically depend on the position of the user within the exoskeleton and the characteristics of the control law.
\end{abstract}

Methods: The present study aims at improving WS of the upper limb by providing a weight model considering joint misalignments and a control law including feedforward terms learned from a prior population-based analysis. Three experiments are respectively conducted on 29, 17 and 19 participants who performed posture maintenance and pointing movements with the forearm in the sagittal plane. The first two experiments were used to build an accurate WS control law and the third experiment was conducted to compare the effects of different WS control laws on human movement and assess their quality. During these three experiments, kinematic data and eletromyographic activity of elbow flexors and extensors were measured. Interaction forces were measured with a force/torque sensor placed between the human segment and the robot link.

Results: The introduction of joint misalignments in the WS model allowed to drastically reduce the model errors in terms of weight estimation. The use of a feedforward architecture based on model and errors learned during experiments, coupled to a force feedback, allowed to reduce model tracking errors in both static and dynamic conditions during vertical movements, which induce substantial variations of gravitational torques. Overall, WS did not affect the general kinematic motion parameters of the participants and decreased the activity of antigravity muscles (flexors). However, WS increased the activation of extensors because weight is usually exploited by humans to accelerate a limb downward.

Conclusion: A new weight compensation model considering joint misalignments was introduced and data showed their prominent role on WS accuracy and homogeneity. Three WS control laws were compared and results indicated that classical control methods were not sufficient to provide an accurate tracking of the weight model during dynamic vertical movements but that introducing simple feedforward terms learned from previous measures could significantly improve WS accuracy. Accordingly, WS reduced significantly activity in flexors in both static and dynamic conditions. Nevertheless, WS tended to increase the activity of extensors, which might be an important factor in a rehabilitation perspective. Indeed, if the present WS control law will be very helpful to allow patients accelerating the arm upward despite some muscle weakness, it may have an opposite effect when accelerating the arm downward. A partial WS controller could thus be more appropriate in rehabilitation applications.

Keywords: Weight support; Rehabilitation robotics; Exoskeleton control; Human/Robot interaction; Human identification 


\section{Background}

Active exoskeletons are promising devices in many areas and their potential benefits in various applications have been extensively studied in the past decades. In particular, active exoskeletons have been tested as a solution to improve rehabilitation processes in stroke patients $[1,2]$. They are expected to help patients to recover more quickly and more permanently their motor functions due to the advantage offered by robotics such as reliability and repeatability [3]. Because of their versatility, active exoskeletons can even be used to help disabled people to perform daily living activities such as walking $[4,5,6]$. All these applications are conditioned by several critical functionalities including transparency [7] and the ability to compensate for the user's weight [8]. Transparency is the ability of an exoskeleton to influence human movement as little as possible when worn [9] and has already been extensively studied $[10,11,12,13,14]$. It is for instance critical at the end of a rehabilitation process to ensure that the robot will not lead the user to adopt abnormal motor patterns. Weight support (WS) has received less attention but can be defined as the fact of compensating both the exoskeleton dynamics (i.e. being transparent) and the weight of the human body segments. Having a robot carrying the human segments is useful to relieve the user from the strong constraints and efforts imposed by gravity and to ease its movements. This function can be very helpful for patients with muscle weakness and critical to rehabilitation protocols as it can increase their motor repertoire [1, 2, 8, 15]. We note that WS has also been used in lifting and carrying tasks in the industrial context in order to decrease the occurrence of musculoskeletal disorders in workers $[16,17]$. In any case, the goal is to reduce the user's muscular efforts related to gravity. Besides studies restricted to the horizontal plane $[18,19,20]$, control strategies for WS in general movements and their impact on the human motion have been rarely studied for the upper limb, except in the case of the hand [21, 22] (see related works in Section 1.2 for details). Indeed, active WS requires building a weight compensation model that includes the estimation of various parameters such as segment mass and location of its center of gravity, which is a long-standing anthropometric issue [23] (see related works in Section 1.1 for details). Therefore, designing accurate in situ identification models and protocols for human masses is the first step for achieving generic WS. Designing efficient control laws is the second step to track the identified

\footnotetext{
${ }^{*}$ Correspondence: dorian.verdel@universite-paris-saclay. fr

${ }^{1}$ CIAMS, Université Paris-Saclay, Orsay, France

Full list of author information is available at the end of the article

tDorian Verdel and Simon Bastide contributed equally to this work
}

weight model during dynamic movements. These two steps are crucial towards an easier integration of exoskeletons with effective WS functionality.

\subsection{Human segments identification}

The reference attempt to measure these parameters was based on data from Chandler and al. [24], collected on six male cadavers (mean age 54). These data led to the creation of anthropometric tables such as De Leva tables [25, 26] and Winter tables [27]. Nevertheless, these tables are notoriously inaccurate and are not adapted to design a personalized compensation. Other methods such as X-rays and tomography analysis have led to better results [23] but are not satisfying in terms of material as they require heavy devices irradiating the participants. Non-invasive approaches based on motion capture analysis emerged with this technology, allowing precise identification of body geometry [28]. Motion capture-based identification techniques were developed to provide results on inertial parameters [29, 30], viscoelastic parameters [31] and can be applied in real-time $[32,33]$ during optimal movements in the identification sense [34]. Most of these experiments were conducted on relaxed (i.e. passive) participants, which was verified by means of electromyographic (EMG) measures. All these techniques still present the major disadvantage of requiring a special room equipped with motion capture technology, which few hospitals and companies can afford. An alternative way to identify human parameters would be an in situ identification using exoskeleton data (such as kinematic or force/torque (FT) measures) and, therefore, avoiding the use of external measurement tools. Fewer studies have been conducted in this perspective. The upper-limb masses identification was carried out with different methods $[8,35]$ suggesting that the most efficient method is to identify the vertical force generated by the segment when the human is completely relaxed, as during certain experiments based on motion capture techniques. This idea of in situ identification was also developed for lower limbs [36]. The force measurement is, in these experiments, either obtained from FT sensors placed at the level of the connection between the human and the robot or from torque sensors placed at the joints of the robot. The main limitation of these studies was the fact that joint misalignments (JM) between the user and the exoskeleton were not considered. Indeed, these misalignments have an impact on the interaction efforts between the user and the exoskeleton [11]. Therefore, they can lead to under or over compensation of weight because of an erroneous identification of the human segment mass. As a consequence, the present paper focuses on introducing a general model of weight taking into account JM for a limb with the objective to generate more accurate compensations. 


\subsection{Weight model tracking}

The second step to achieve an accurate WS is to carefully follow the identified weight model. As previously introduced, WS is a necessary step in many functional rehabilitation processes $[1,2,15]$. Therefore, its effect have been studied on disabled patients often using passive systems. These investigations were oriented towards the analysis of muscle activities in different configurations. In the horizontal plane [37], in the sagittal plane $[38,39]$ and during three-dimensional movements [40,41], a global decrease of electromyographic (EMG) activities and an increase in the number of muscular synergies were observed. Nevertheless, these findings did not address the problem of compensating weight with an active device in the vertical plane. In fact, few studies considered this problem. As previously mentioned, it was discussed for rhythmic hand movements $[21,22]$ using box Jenkins models for motors in order to analyze the stability of human movements under different conditions. Some studies about upper-limb WS with active exoskeletons such as [35, 8] described different compensation methods but did not focus on the associated design of an accurate control law. Furthermore, these studies either considered lightweight limbs as hands or dealt with movements performed in horizontal plane that induce a constant weight torque. WS could even be obtained natively in the horizontal plane without dedicated control with the KinArm exoskeleton [18, 19, 20]. In these cases, only a partial movement set generating few variations in weight torque is considered, which does not induce the problems that occur in more general settings. As a consequence, the present paper will focus on problem of WS for an arm moving in the sagittal plane as this will generate substantial variations in gravitational torques. The objectives are thus to (1) build an accurate weight compensation model in situ for each participant considering JM and (2) design a WS control law that follows this model despite variations of gravitational torques during motion in the vertical plane.

Considering these two main objectives, the general methodology applied in the present study is presented in Figure 1, and the rest of this paper is organized as follows. In Section 2.1 a weight model and its identification procedure are presented. Then, in Section 2.2, the construction of the weight compensation control law is described and some successive solutions are proposed. In Section 2.3 a validation task allowing to compare the different solutions and validate the control law's design methodology is described. All the experimental results are presented in Section 3 and discussed in Section 4.

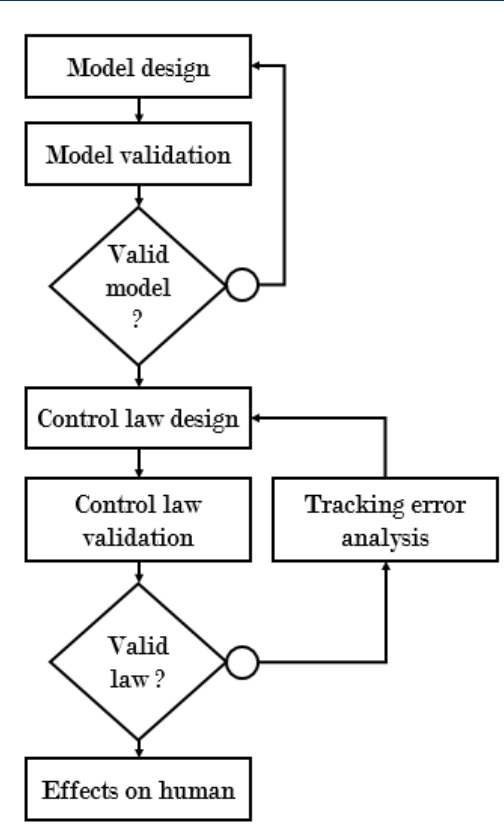

Figure 1: Applied methodology

\section{Methods}

\subsection{Weight model with joint misalignments and} identification

General case: The first step to build a WS control is to identify precisely the mass of the limb used in the movement. Given the fact that JM between the exoskeleton and the user are inevitable [11], these misalignments must be identified to build a homogeneous weight compensation. This has not yet been explored in previous weight compensation studies as the assumption of perfect alignment between the user and the exoskeleton is often implicitly made. In the present section, a general model characterizing JM and their influence on weight expression for one revolute robot link and its associated human segment is described. This model could be applied to multiple limbs by adding misalignments and angular parameters. In the general case, misalignments between a human segment and an exoskeleton link can be described by three rotations and three translations as presented in Figure 2a. As demonstrated in [8], it is more efficient to compensate weight based on a vertical projection of the measured efforts (in an absolute frame) with a FT sensor placed at the level of the interaction, thus not using pre-established anthropometric tables to build the weight model. Therefore, the three translations between the human and the robot joints do not have any impact in terms of weight compensation, as long as the human-exoskeleton connection is compliant. Indeed, if the connections are not compliant enough, residual 
forces due to the hyperstatic nature of the physical connection between the robot and the human may appear and bias the weight model [11].

The dimension of the orientation problem can be reduced by choosing a proper reference frame taking advantage of the fact that only the orientation of a limb in a vertical plane has an impact on the resulting weight compensation to apply. Indeed, the three elementary rotations involved in the orientation of the human segment and the three ones involved in the orientation of the robot link can be reduced to two rotations if the objective is to compensate weight. To achieve this reduction, the reference frame can be defined with a vertical $\mathbf{y}_{\mathbf{0}}$ axis inducing the other components as described in Equation (1),

$$
\left\{\begin{array}{l}
\mathrm{z}_{0}=\frac{\mathrm{x}_{\mathrm{r}} \times \mathrm{y}_{0}}{\left\|\mathrm{x}_{\mathrm{r}} \times \mathrm{y}_{0}\right\|} \\
\mathrm{x}_{\mathbf{0}}=\mathrm{y}_{0} \times \mathrm{z}_{0}
\end{array}\right.
$$

where $\mathbf{x}_{0}$ and $\mathbf{z}_{\mathbf{0}}$ are the two other world frame vectors and $\mathbf{x}_{\mathbf{r}}$ is the vector supporting the robot link as described in Figure 2a. The reference frame origin can then be defined as $O=O_{r}$, where $O_{r}$ is the origin of the robot link frame. This frame containing the robot link in a vertical plane $\left(O, \mathbf{x}_{\mathbf{0}}, \mathbf{y}_{\mathbf{0}}\right)$ can always be defined, without any assumption regarding the robot limb orientation. In this frame the orientation of the robot link is only characterized by one rotation as described in Equation (2),

$$
\mathbf{x}_{\mathbf{r}}=R_{r 0}\left(\mathbf{z}_{\mathbf{0}}, \phi_{r}\right) \mathbf{x}_{\mathbf{0}}
$$

where $R_{r 0}\left(\mathbf{z}_{\mathbf{0}}, \phi_{r}\right)$ is the rotation of angle $\phi_{r}$ around $\mathbf{z}_{\mathbf{0}}$ characterizing the orientation of the robot link in the previously defined vertical plane. This rotation is always known as it only depends on the robot's angular positions and its geometry. In the rest of the present paper, all rotations will be noted $R_{a b}(\mathbf{v}, \theta)$, where $R_{a b}$ is the rotation from frame $b$ to frame $a, \mathbf{v}$ is the vector supporting rotation axis and $\theta$ is the angle of rotation. As a result, the robot frame can be defined as described in Equation (3),

$$
\left\{\begin{array}{l}
\mathbf{y}_{\mathbf{r}}=R_{r 0}\left(\mathbf{z}_{\mathbf{0}}, \phi_{r}\right) \mathbf{y}_{\mathbf{0}} \\
\mathbf{z}_{\mathbf{r}}=R_{r 0}\left(\mathbf{z}_{\mathbf{0}}, \phi_{r}\right) \mathbf{z}_{\mathbf{0}}=\mathbf{z}_{\mathbf{0}}
\end{array}\right.
$$

In this robot link frame, the orientation of the vector supporting the human segment $\mathbf{x}_{\mathbf{h}}$ defined in Figure 2a is defined by two successive rotations. A first rotation to define $\mathbf{y}_{\mathbf{h}}=\mathbf{y}_{\mathbf{h}, \mathbf{p}}$ and an intermediate $\mathbf{x}_{\mathbf{h}}$ (called $\mathbf{x}_{\mathbf{h}, \mathbf{p}}$ as defined in Figure 2a), followed by a rotation to define $\mathbf{x}_{\mathbf{h}}$ as described in Equation (4),

$$
\left\{\begin{aligned}
\mathbf{y}_{\mathbf{h}, \mathbf{p}} & =R_{h r, 1}\left(\mathbf{z}_{\mathbf{0}}, \phi_{h}\left(\phi_{r}\right)\right) \mathbf{y}_{\mathbf{r}} \\
\mathbf{x}_{\mathbf{h}, \mathbf{p}} & =R_{h r, 1}\left(\mathbf{z}_{\mathbf{0}}, \phi_{h}\left(\phi_{r}\right)\right) \mathbf{x}_{\mathbf{r}} \\
\mathbf{y}_{\mathbf{h}} & =\mathbf{y}_{\mathbf{h}, \mathbf{p}} \\
\mathbf{x}_{\mathbf{h}} & =R_{h r, 2}\left(\mathbf{y}_{\mathbf{h}, \mathbf{p}}, \psi_{h}\left(\phi_{r}\right)\right) \mathbf{x}_{\mathbf{h}, \mathbf{p}}
\end{aligned}\right.
$$

where $\mathbf{x}_{\mathbf{h}}$ and $\mathbf{x}_{\mathbf{r}}$ are the vectors supporting the human segment and the robot link, respectively. The two human orientation parameters might not be independent from the robot link orientation, therefore they are defined as functions of $\phi_{r}$. The projection of the so-defined $\mathbf{x}_{\mathbf{h}}$ in the plane $\left(O, \mathbf{x}_{\mathbf{0}}, \mathbf{y}_{\mathbf{0}}\right)$ reduces the orientation of the human segment to the single $\phi_{h}\left(\phi_{r}\right)$ rotation. This projection is possible because the rotation $R_{h r, 2}\left(\mathbf{y}_{\mathbf{h}, \mathbf{p}} \psi_{h}\left(\phi_{r}\right)\right)$ does not affect the weight measured in the robot link frame as it does not impact the human segment orientation in the previously defined vertical plane. Eventually, the weight of the human segment can be characterized in the same vertical plane as described in Equation (5),

$$
\mathbf{W}_{\mathbf{h}}^{\mathbf{h}}=\mathbf{W}_{\mathbf{h}}^{\mathbf{h}, \mathbf{p}}=R_{h 0}\left(\mathbf{z}_{\mathbf{0}}, \phi_{r}+\phi_{h}\left(\phi_{r}\right)\right)^{T} \mathbf{W}_{\mathbf{h}}^{\mathbf{0}}
$$

where $\mathbf{W}_{\mathbf{h}}^{\mathbf{h}}$ and $\mathbf{W}_{\mathbf{h}}^{\mathbf{0}}$ are the human segment weight expressed in the human and in the world frame, respectively. The rotation $\phi_{r}$ is already defined and $\phi_{h}\left(\phi_{r}\right)$ is the previously defined angle of rotation between the robot and the human segment around $\mathbf{z}_{\mathbf{r}}$. The projected human segment is represented by the axis $\left(O_{h, p}, \mathbf{x}_{\mathbf{h}, \mathbf{p}}\right)$ on Figure 2a. The expression of the weight in the human frame is necessary to compute the gravitational torque at the level of the joint and, therefore, to compute the weight compensation to be applied by the robot.

As this reduction of the problem to a planar problem is always possible by projecting $\mathbf{x}_{\mathbf{h}}$ in the plane $\left(O, \mathbf{x}_{\mathbf{0}}, \mathbf{y}_{\mathbf{0}}\right)$, the rest of the present study will focus on planar human movements in the sagittal plane. More precisely, we will focus on accurately identifying and compensating the weight of a human forearm and hand in the sagittal plane during goal-directed movements. All rotation operators are detailed in appropriate basis in Appendix.

Sagittal plane case study: In the present study, a modified ABLE exoskeleton is used (see Section 2.3.1 for detailed presentation). This exoskeleton is modified to be as compliant as possible in terms of connection to the user. More specifically, residual torques and forces due to position and orientation errors between the exoskeleton and the user are removed by a 


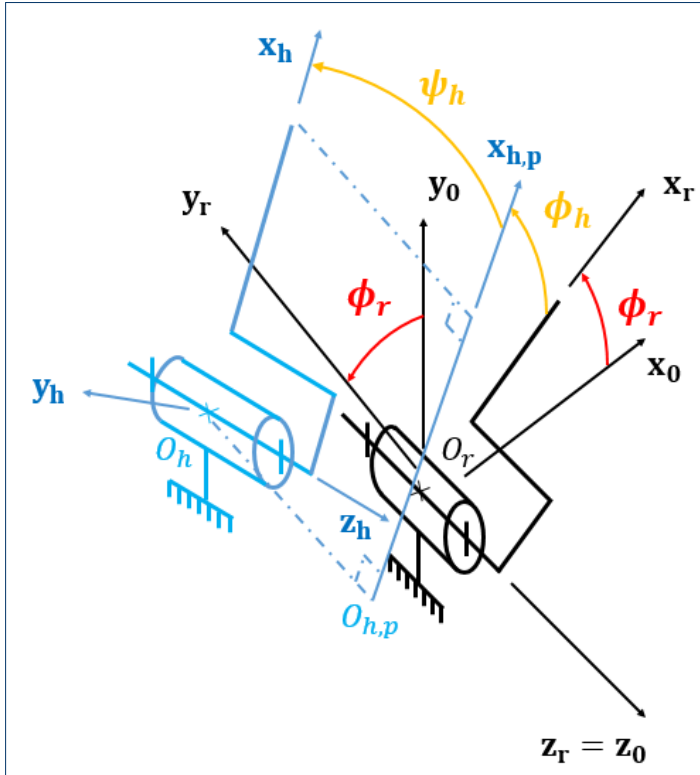

(a)

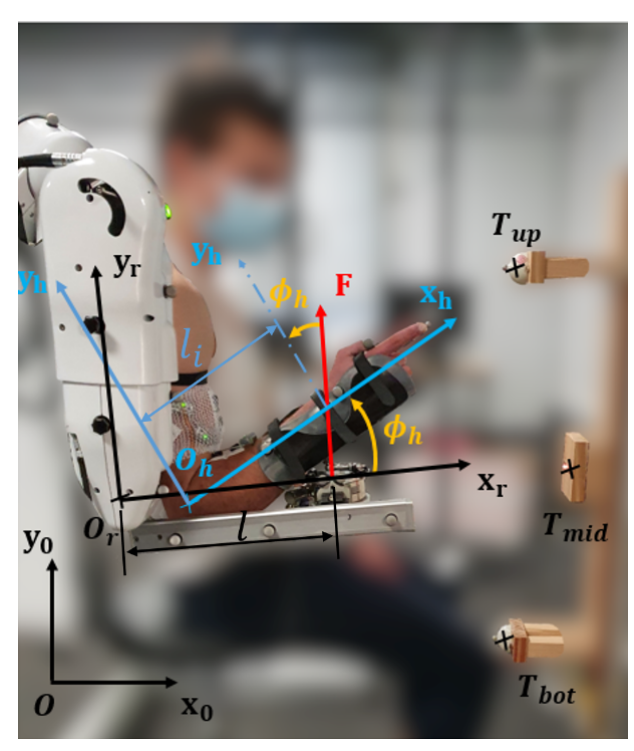

(b)

Figure 2: Misalignments between human and exoskeleton. (a) General situation. (b) Sagittal plane case study.

ball joint coupled to a prismatic joint (i.e. a slider on a rail), which aims at improving the quality of the interaction in terms of comfort. These modifications imply that measures of the FT sensor along $\mathbf{x}_{\mathbf{r}}$ are not exploitable. Therefore, our WS control can only rely on the local normal measure of the sensor along $\mathbf{y}_{\mathbf{r}}$ in this case study which is a slight difference with the method proposed in [8]. In this situation, the rotation previously defined by $\phi_{r}$ is directly equal to the rotation induced by a robot motor, therefore we have $\phi_{r}=q$, where $q$ is the angular position of the robot elbow. The weight model (i.e. the evolution of the weight torque $\tau_{h}$ with regard to $q$, the angular position of the robot with a horizontal reference) that should be identified is a particular case of Equation (5) and is described in Equation (6),

$$
\begin{aligned}
\tau_{h} & =\left(x_{g} \mathbf{x}_{\mathbf{h}} \times \mathbf{W}_{\mathbf{h}}\right) \cdot \mathbf{z}_{\mathbf{0}} \\
& =-m_{f, h} g x_{g} \cos \left(q+\phi_{h}(q)\right)
\end{aligned}
$$

where $\tau_{h}$ is computed under the assumption that the center of mass of the human segment is on the axis $\left(O_{h}, \mathbf{x}_{\mathbf{h}}\right)$ (as in [8]), $m_{f, h}$ is the addition of the masses of the forearm, the hand of the user and the connection to the robot that must be identified, $x_{g}$ is the center of mass position in the human frame, $q$ is the angular position of the robot elbow (measured by incremental encoders) and $\phi_{h}(q)$ is the misalignment between the robot and the user forearm segments that must be identified. The resulting force interaction model that must be applied by the robot to compensate for the weight is obtained through equilibrium of torques and described in Equation (7) by

$$
F=m_{f, h} \frac{x_{g}}{l_{i}} g \frac{\cos \left(q+\phi_{h}(q)\right)}{\cos \left(\phi_{h}(q)\right)}
$$

where $F$ is the opposite of the normal interaction force measured by the FT sensor and $l_{i}$ is the distance between the human elbow and the interaction point along $\mathbf{x}_{\mathbf{h}}$. As the physical interface with the robot completely envelopes the human forearm, the interaction point is modeled as a point belonging to the axis $\left(O_{h}, \mathbf{x}_{\mathbf{h}}\right)$. Note that this interaction force model is only valid for static situations where inertial and viscous terms and human muscles activation does not exist or is negligible. As a consequence, this model can only be identified on static positions of the robot and with a relaxed user as it is often the case when trying to compensate for weight $[36,35,8]$. It is interesting to note that using a force equilibrium seems to be inappropriate in this situation. Indeed, it would require to neglect the interactions at the level of the human joint to solve the problem, which amounts to use a classical weight model without JM.

By design, the torque at the robot elbow joint resulting from this weight compensation model is equal to the interaction force of Equation (7) multiplied by the lever arm between the robot elbow joint center $O_{r}$ 
and the direction of this force, which corresponds to the distance between $O_{r}$ and the slider (see Figure 2b). This is described by Equation (8),

$$
\begin{aligned}
\tau_{i} & =l F \\
& =l m_{f, h} \frac{x_{g}}{l_{i}} g \frac{\cos \left(q+\phi_{h}(q)\right)}{\cos \left(\phi_{h}(q)\right)}
\end{aligned}
$$

where $\tau_{i}$ is the resulting interaction torque to apply for weight compensation and $l$ is the distance between $O_{r}$ and the slider.

Model without JM: For comparison purposes, the model without JM is defined below. As JM are described by $\phi_{h}$ in our framework, the case $\phi_{h} \triangleq 0$ corresponds to a model without JM and is described by Equation (9),

$$
\tau_{i, w j m}=l m_{f, h} \frac{x_{g}}{l_{i}} g \cos (q)
$$

where $\tau_{i, w j m}$ is the torque to apply to compensate for weight without JM.

Optimization problem: The one degree of freedom weight model presented in Equation (7) can be adapted to formulate an optimization problem in the least squares sense. In the rest of the present study, only a zero-order approximation of $\phi_{h}(q)$ (i.e. $\left.\phi_{h}(q) \approx \phi_{h}\right)$ is considered as experiments tends to show that this approximation is reasonable on our tested movement ranges (see Section 3.1). This approximation might not hold for movements of large amplitude (typically for $\phi_{h}+q>40^{\circ}$ and $\phi_{h}+q<-60^{\circ}$ in our case). This is not a problem for the future generalization as a more complex model of $\phi_{h}(q)$ could be identified through experiment. The resulting optimization problem is presented in Equation (10),

$$
\left(\begin{array}{c}
\hat{m}_{e q, h} \\
\hat{\phi}_{h}
\end{array}\right)=\underset{m_{e q, h}, \phi_{h}}{\arg \min }\left(F-m_{e q, h} g \frac{\cos \left(q+\phi_{h}\right)}{\cos \left(\phi_{h}\right)}\right)^{2}
$$

where $\hat{m}_{e q, h}$ and $\hat{\phi}_{h}$ are the estimated values of $m_{e q, h}$ and $\phi_{h}$, respectively, and $m_{e q, h}=m_{f, h} x_{g} / l_{i}$. In order to accurately estimate these two values, as many measures of $F$ and $q$ as possible must be collected for each participant during a static and passive experiment. Therefore, the identification procedure detailed below is carried out.
Participants: The evaluation of the present approach was performed with 17 healthy right-handed participants. Basic anthropometric characteristics of the participants were as follows: 7 females, 10 males, mean weight $70.7 \pm 9.7 \mathrm{~kg}$, mean height $176 \pm 7.8 \mathrm{~cm}$, mean age $24 \pm 4.9$ years old, mean forearm length $25.5 \pm 1.4 \mathrm{~cm}$ and mean hand length $18.6 \pm 1.3 \mathrm{~cm}$. It should be noted that limb lengths were measured approximately with a measuring tape. Therefore, results in terms of limb length are not exploitable as relevant anthropometric data but still allow us to have a first approximation of the population on which the control laws have been tested. A written informed consent was given by each participant as required by Helsinki declaration [42]. The experimental protocol was approved by the ethical committee for research (Université Paris-Saclay, 2017-34). The written consent and the approval of the protocol were also obtained for all the experiments presented in the rest of the present paper.

Identification protocol: As stated previously, the identification of the human arm's parameters is performed under static conditions and with the participant being as passive as possible. Participants were placed with their shoulder at the intersection of the first three joints of ABLE. They were connected to ABLE at the level of their forearm and arm (see Section 2.3 for details). Participants were asked to relax while the elbow joint of the robot moved slowly between 20 positions equally spread across its whole work-space: $\left[-85.94^{\circ}, 39.53^{\circ}\right]$. Each position was statically maintained during $5 \mathrm{~s}$. Measures from electromyographic (EMG) sensors were used to check in real-time that the participant was relaxed. Angular positions of the robot and interaction forces were measured at $1 \mathrm{kHz}$. The resolution of the optimization problem presented in Equation (10) was then carried out using the "lsqnonlin" function from the "Optimization Toolbox" of Matlab(C). For comparison purposes, the same optimization was carried out with $\phi \triangleq 0$ (only the observed mass was identified in this case).

Model identification error: After identification of the model, the mean absolute error (MAE) between predictions and measures was computed for each subject and both models (with and without JM) as expressed in Equation (11)

$$
M A E=\frac{1}{N} \sum|\hat{F}(q, \phi)-F|
$$

where $N$ is the number of samples, $\hat{F}(q, \phi)$ is the predicted force resulting from model identification and $F$ is the measured force. 
Statistical analysis: A pairwise $t$-test comparison was conducted between the two sets of errors using the Pingouin package [43]. The significance level was fixed at $p<0.05$.

Adjustable compensation: The WS level can be adjusted easily by adjusting the model presented in Equation (8). Indeed, adding a real coefficient to the weight model allows to choose directly the level of the desired compensation. The modified model is expressed in Equation (12),

$$
\tau_{i}=\alpha l m_{e q, h} g \frac{\cos \left(q+\phi_{h}\right)}{\cos \left(\phi_{h}\right)}
$$

where $\alpha$ is the introduced coefficient. For example, during the course of rehabilitation, $\alpha$ can be varied between 1 (for a complete WS) and 0 (for a transparent behavior at the end of a therapy). It should be noted that the human parameters must be identified before changing the compensation level.

Achieving an effective WS requires the ability to accurately track the model defined in Equation (8), which will be the main focus of the rest of Section 2 .

\subsection{Weight support control laws}

This section describes the off-line construction of a general WS control law based on compensation errors observed during experiments. In other terms, the underlying learning process necessary to design the control laws is described in the current section.

Initial force feedback control law: All the control laws used in the present study are based on an accurate compensation of the robot dynamics. This compensation is based on the methods described in [14]. Over this dynamic compensation of the robot, the FT sensor is used to build a feedback control based on the normal interaction forces with the user [44]. In classical approaches, the control is often assured by a partial or complete Proportional Integral Derivative (PID) corrector. The parameters of this type of corrector are system-dependent and task-dependent. In our case a PI corrector was implemented as the derivative coefficient generated instabilities. The control law resulting from the classical PI approach is described by Equation (13),

$$
\begin{aligned}
& \tau_{c t r l}=K_{p}\left(\hat{\tau}_{i}(q, \phi)-\tau_{i}\right) \\
& \quad+K_{i} \int_{0}^{t}\left(\hat{\tau}_{i}(q, \phi)-\tau_{i}\right) d t+\hat{\tau}_{\text {comp }}(\ddot{q}, \dot{q}, q)
\end{aligned}
$$

where $\tau_{c t r l}$ is the control torque to apply at the elbow joint of the robot, $\hat{\tau}_{i}(q, \phi)$ is the estimated WS control torque resulting from Equation (8), $\tau_{i}$ is the measured interaction torque resulting from FT sensor measurements and $\hat{\tau}_{\text {comp }}(\ddot{q}, \dot{q}, q)$ is the estimated compensation of the robot dynamics based on the model presented in [14] and in Equation (14). This model is dependent on $\ddot{q}$ (respectively $\dot{q}$ and $q$ ) the angular acceleration (respectively angular velocity and position) of the elbow joint. The angular velocity and acceleration are estimated by numerical differentiation of the angular positions of the robot. The angular acceleration thereby estimated is too noisy to be used in the exoskeleton control, therefore, inertial terms are not taken into consideration in the dynamic compensation. The control scheme describing this behavior is presented in Figure 4 in black. This law will be called BC (for Basic Compensation) in the rest of the present paper. The compensation of robot dynamics is described by Equation (14),

$$
\hat{\tau}_{\text {comp }}(\ddot{q}, \dot{q}, q)=\hat{\mathcal{M}}(q) \ddot{q}+\hat{\mathcal{C}}(q, \dot{q})+\hat{\mathcal{G}}(q)+\operatorname{sign}(\dot{q}) \hat{\tau}_{C}+\hat{\nu} \dot{q}
$$

where $\hat{\mathcal{M}}(q)$ is the estimated inertia of the segment, $\hat{\mathcal{C}}(q, \dot{q})$ is the estimated Coriolis/centrifugal efforts, $\hat{\mathcal{G}}(q)$ is the estimated gravitational torque induced by the robot weight, $\hat{\tau}_{C}$ is the estimated Coulomb dry friction torque, $\hat{\nu}$ is the estimated viscous friction coefficient and $\hat{\tau}_{\text {comp }}$ is the estimated motor torque of the robot used to compensate for the robot dynamics.

Feedforward term dependent on theoretical interaction force: As it will be seen in the results, the initial force-feedback control law was insufficient to ensure an efficient tracking of the theoretical model during vertical arm movements (see Section 3.2 for details). The analysis of measured interaction forces during experiments with this classical approach allowed us to uncover that model tracking errors depended on the magnitude of the theoretical force with an affine trend (see Figure 3a).

This trend revealed by the experiments allows to add a feedforward term to the previous control law, based on a linear model extracted from errors analysis. This term is defined by Equation (15) as follows:

$$
\tau_{F F, \tau}=K_{F F, \tau} \hat{\tau}_{i}(q, \phi)+\tau_{F F, \tau 0}
$$

where $\tau_{F F, \tau}$ is the feedforward torque added to the control law presented in Equation (13), $K_{F F, \tau}$ is the slope of the identified linear model of Figure $3 \mathrm{a}, \hat{\tau}_{i}(q, \phi)$ 
is the theoretical torque resulting from Equation (6) and $\tau_{F F, \tau 0}$ is the Y-intercept of the identified linear model of Figure 3a multiplied by $l$. The approach consists in learning the error behavior across trials and participants to improve the overall WS quality, by adding this linear model as a feedforward torque. This learned model is represented in blue in Figure 4 and in Equation (17). The law taking into account this model will be called FFTF (for Feedforward Force based on Theoretical Force) in the rest of the present paper.

Feedforward term dependent on the robot angular position: Despite significant improvements in terms of tracking error, it will be seen that the previous feedforward approach still generated too much tracking errors and was still inadequate to ensure an efficient tracking of the theoretical model (see Section 3.2 for details). The analysis of measured interaction forces during experiments on the previous approach allowed us to exhibit another mean behavior of the compensation errors presented in Figure 3b, following the learning approach introduced previously.

The behavior exhibited by the experiments allows to complete our feedforward approach by adding another linear compensation depending on the angular position of the robot. This model is described by Equation (16),

$$
\tau_{F F, q}=K_{F F, q} q+\tau_{F F, q} 0
$$

where $\tau_{F F, q}$ is the second feedforward torque added to the control law presented in Equation (13), $K_{F F, q}$ is the slope of the identified linear model of Figure $3 \mathrm{~b}, q$ is the angular position of the robot and $\tau_{F F, q} 0$ is the Y-intercept of the identified linear model illustrated in Figure 3b. The measures given in Figure $3 \mathrm{~b}$ do not suggest the use of a linear model at first sight. Nevertheless, for point-to-point movements between various targets, their pseudo-sinusoidal form is mainly due to delay and lack of inertia compensation in the control laws. These errors could be compensated with intention detection, but this is not the focus of the present study. The learned model is represented in red in Figure 4 and in Equation 17). This law will be called FFCM (for Feedforward Force Complete Model) in the rest of the present paper. The complete WS control law thereby constructed is described by Equation (17),

$$
\begin{aligned}
\tau_{c t r l} & =K_{p}\left(\hat{\tau}_{i}(q, \phi)-\tau_{i}\right) \\
& +K_{i} \int_{0}^{t}\left(\hat{\tau}_{i}(q, \phi)-\tau_{i}\right) d t+\hat{\tau}_{c o m p}(\dot{q}, q) \\
& +\tau_{F F, \tau}+\tau_{F F, q}
\end{aligned}
$$

Individualized compensation: It should be noted here that in a rehabilitation context, it would be possible to learn the FFTF and FFCM control laws for only one individual and, therefore, build a fully personalized control. Indeed, the process of learning errors requires an amount of data that can be obtained on only one individual. As different levels of theoretical forces are needed, changing the level of applied WS (by modifying $\alpha$ from Equation (12), in Section 2.1) is a solution to identify the parameters of the FFTF law. The identification of the parameters of a personalized FFCM would also be possible as it would only require to perform a small number of upward and downward movements on a large amplitude (such as $60^{\circ}$ ). Personalized learning could also be performed on-line contrary to our general learning that requires to gather data from different participants.

Control modes: Two control modes are used in our experiments, the first one is a "transparent" control. In this mode, the control is designed to minimize the normal interaction force (i.e. minimize $F$ ). This "transparent" control mode is used as a baseline condition to quantify alterations in the human movement when submitted to different WS controls.

The second control mode corresponds to a classical WS where the robot should compensate its own dynamics and the weight of the forearm/hand of the user during movements in the sagittal plane. Therefore, the robot is controlled to maintain a normal interaction force that follows the model presented in Equation (6). The three previous control laws (i.e. BC, FFTF and FFCM) are tested on this control mode and their respective capacities in terms of model tracking are tested and compared in Section 3.2.

\subsection{Design of the experimental validation of the control laws}

2.3.1 Materials and task

Participants As described in Section 2.2, three control laws are tested sequentially. The second control law (FFTF) was constructed from data recorded with the first law (BC) and the third control law (FFCM) was constructed with data recorded when using the second control law (FFTF). Therefore, three sessions were necessary to build and test our three control laws. Data relative to the participants of each session are reported in Table 1 and in Table 2.

Certain participants were involved in the three sessions ( $N=8$ participants). Participants were all naive about the purpose of the experiment. As our goal is to build a control law that can be used by different individuals, most participants from the first two sessions were not the same. 


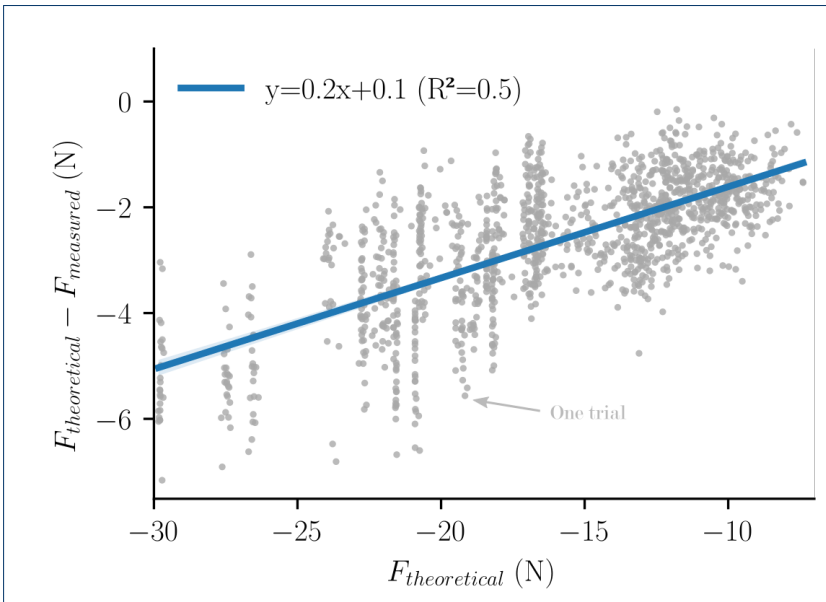

(a)

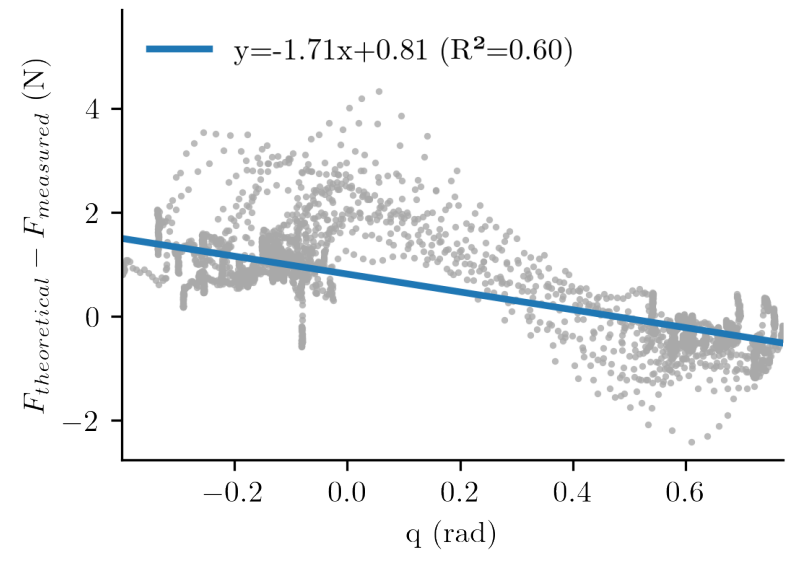

(b)

Figure 3: Mean linear models extracted from experiments. (a) Interaction force versus theoretical force and mean linear behavior. (b) Interaction force versus angular position and mean linear behavior.

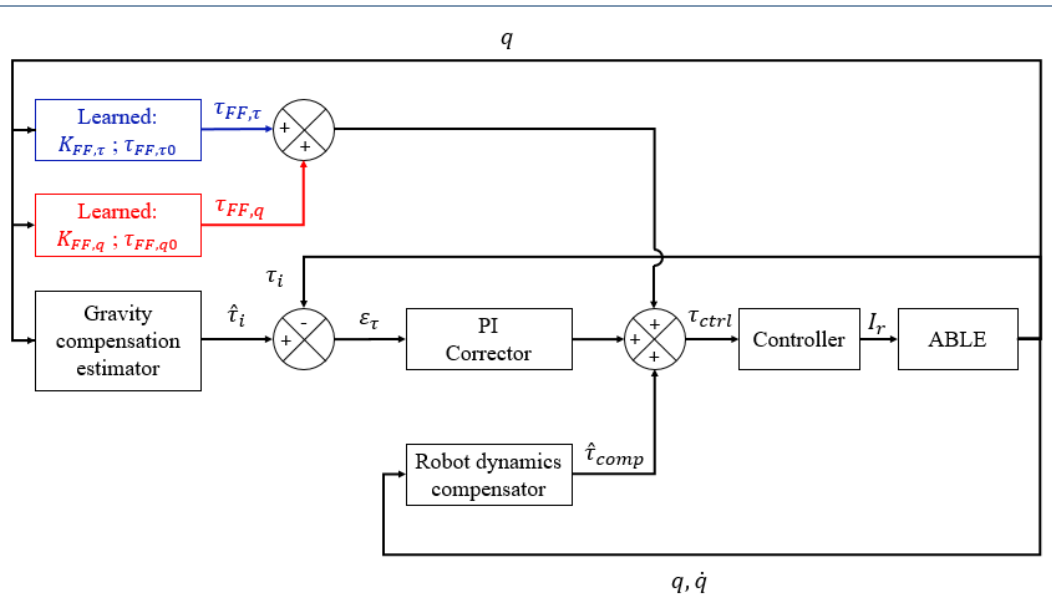

Figure 4: Control schemes used during the present study. Black: basic PI control loop and weight torque estimation. Blue: FFTF term. Red: FFCM term.

\begin{tabular}{cccccccc} 
Session & $\mathrm{N}$ & Sex & Age (years) & Weight $(\mathrm{kg})$ & Height $(\mathrm{cm})$ & $\mathrm{FL}(\mathrm{cm})$ & $\mathrm{HL}(\mathrm{cm})$ \\
\hline 1 & 29 & $10 \mathrm{~F} ; 19 \mathrm{M}$ & $23.2 \pm 3.0$ & $67.1 \pm 11.8$ & $174.7 \pm 7.6$ & $25.5 \pm 1.6$ & $18.8 \pm 1.0$ \\
2 & 17 & $7 \mathrm{~F} ; 10 \mathrm{M}$ & $24 \pm 4.9$ & $70.7 \pm 9.7$ & $176 \pm 7.8$ & $25.5 \pm 1.4$ & $18.6 \pm 1.3$ \\
3 & 19 & $6 \mathrm{~F} ; 13 \mathrm{M}$ & $24.3 \pm 2.2$ & $70.7 \pm 9.8$ & $176 \pm 8.4$ & $26.4 \pm 2.0$ & $19.5 \pm 1.2$ \\
\hline
\end{tabular}

Table 1: Data relative to the participants: $N$ is the number of participants, FL is the forearm length and HL is the hand length.

\begin{tabular}{ccc} 
Session & $m_{e q, h}(\mathrm{~kg})$ & $\phi_{h}(\mathrm{rad})$ \\
\hline 1 & $2.13 \pm 0.56$ & $0.27 \pm 0.12$ \\
2 & $1.93 \pm 0.26$ & $0.21 \pm 0.07$ \\
3 & $1.90 \pm 0.36$ & $0.23 \pm 0.07$
\end{tabular}

Table 2: Identified masses and JMs for each session.

ABLE exoskeleton The present study is achieved with an ABLE upper-limb exoskeleton (see FigurE 5a) which has four actuated joints and one free slider whose position and velocity cannot be directly monitored.

The first three actuated joints of ABLE correspond to the three degrees of freedom of the human gleno- 


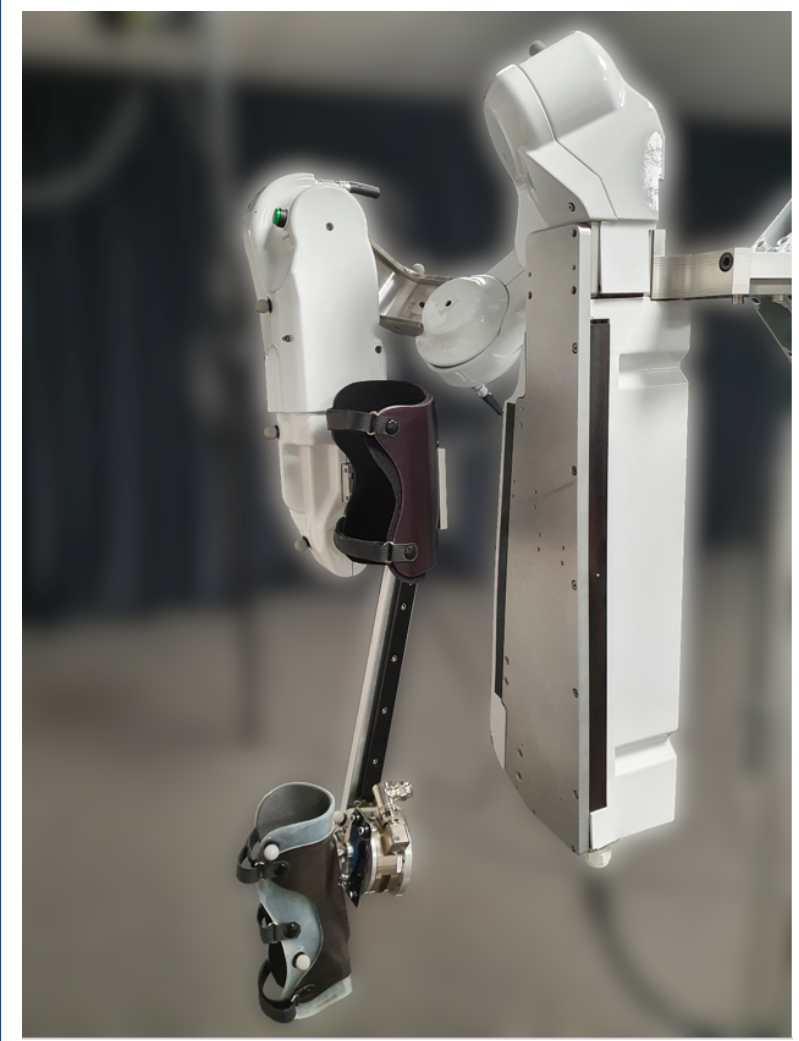

(a)
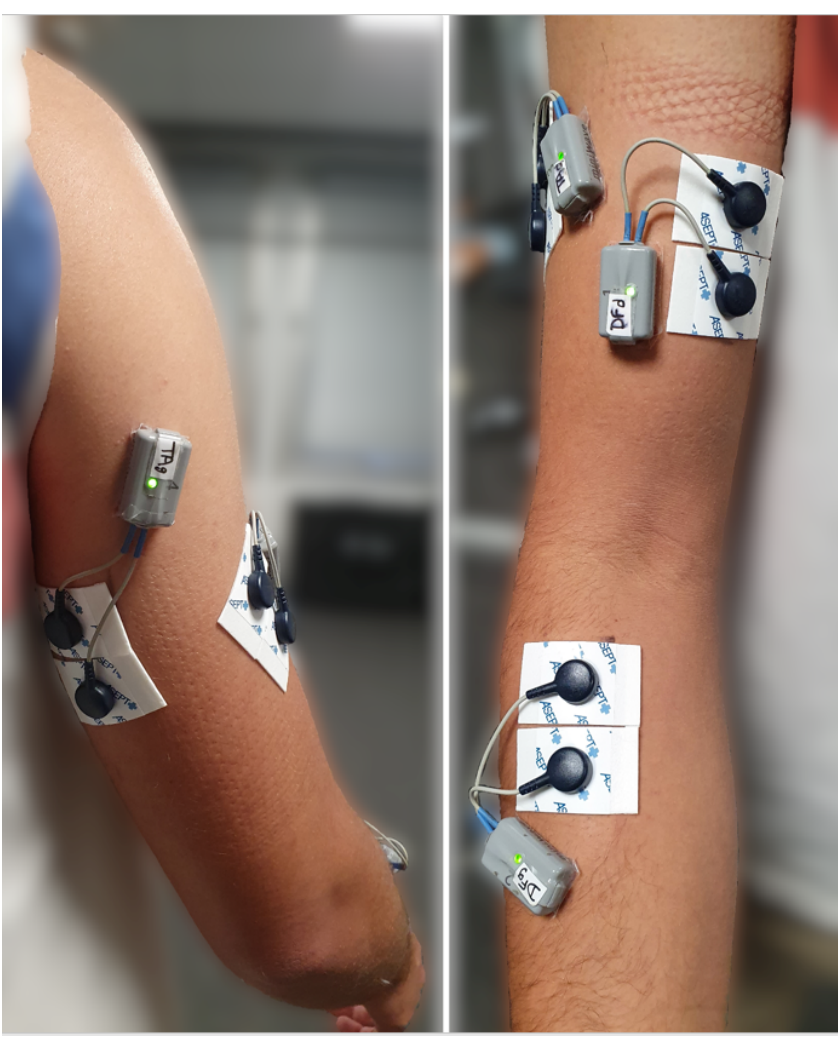

(b)

Figure 5: Material and experimental set-up. (a) ABLE exoskeleton used in the present study. (b) Disposition of EMG sensors (back on left, front on right).

humeral joint (abduction/adduction, internal/external rotation and flexion/extension) and the last actuated joint correspond to the flexion/extension of the human elbow. The slider is moving on a rail, which forms a prismatic joint, and has been added to prevent the occurrence of hyperstatic forces at the level of the connection between ABLE and the user. Furthermore, a ball joint was added between the slider and the connection to the user with the same purpose and, therefore, provide a more ergonomic interaction [11]. For the same purpose, connections are made of a deformable material to adapt to the shape of the participant's limb. The forearm connection is also made to impeach wrist movements in order to ensure that movements are only performed by elbow flexion/extension. This robot was designed to be highly backdriveable and compliant in order to maximize the human/exoskeleton symbiosis [45, 46]. In addition to classical measures of motor positions obtained in realtime by incremental encoders, a FT sensor (1010 Digital FT, ATIC, sample rate: $7 \mathrm{kHz}$ ) was added at the level of the connection between ABLE and the user to measure the interaction forces and provide the feedback for WS control laws.

Other materials Kinematic characteristics of movements were extracted from the robot position data. Participant's muscular activities were measured with four EMG sensors (Wave Plus wireless EMG system, Cometa). These EMGs were placed on two elbow flexor muscles (brachio-radialis and biceps brachii) and two elbow extensor muscles (triceps brachii long head and lateral head). The EMG sensors are placed according to SENIAM recommendations [47] (see Figure 5b).

Motor task As previously motivated (see Section 1 and Section 2.1), the task involved only elbow flexions and extensions. Movements consisted in point-to-point reaching in the sagittal plane. Targets are described in Figure $2 \mathrm{~b}$ as $T_{u p}, T_{b o t}$ and $T_{m i d}$. For the participants, these targets are represented by 3 LEDs. The middle LED is the initial target. The two other LEDs are successively illuminated so as to generate movements of an amplitude of $60^{\circ}$ centered around a horizontal 
axis. Participants are asked to move toward the illuminated LED. The LED are illuminated during $1 s$ to induce a movement duration that stays around $600 \mathrm{~ms}$. Movements are performed under two different conditions, in "transparent" mode and in WS mode $(\alpha=1)$. Between each single point-to-point movement, participants maintained their position during approximately $2 s$.

Participants are placed with their shoulder at the intersection of the first three joints of ABLE's shoulder. They are connected to ABLE at the level of their forearm and arm with large splints to maximize the interaction quality [11] (see Figure 5a).

In the first session, each participant was asked to perform 7 blocks of 30 movements (15 upward and 15 downward), thereby giving a total of 210 movements per participant. The first block was performed in "transparent" (TR) mode to help the participant to learn the task, then 6 blocks were performed under WS with the BC control law. In the second session, each participant performed the 6 WS blocks with the FFTF control law.

In the final validation experiment, 4 blocks of 30 movements are carried out (15 upward and 15 downward), which yielded a total of 120 movements per participant. The first block was also in "transparent" mode for familiarization and then each block was performed with the BC, FFTF or FFCM control law respectively. These three control laws were randomly assigned to one block. At the end of each block, each participant was asked to point towards each target $\left(T_{u p}\right.$, $T_{\text {bot }}$ and $\left.T_{m i d}\right)$ and maintain position for five seconds in order to obtain static measurements.

In order to prevent fatigue effects, 3 minutes pauses were taken between blocks. Note that the performed movements were not particularly demanding due to the presence of a WS (although imperfect) in most conditions.

\subsubsection{Data processing}

Electromyography: EMG signals were filtered using a band-pass filter (fourth-order Butterworth, $[20,450] \mathrm{Hz}$ cut-off frequency), centered and rectified. Signals were normalized by the maximal activity found during the experiment for each subject and each muscle. The envelope of the EMG signal was obtained by applying a low-pass filter (10 Hz cut-off frequency, fifth-order Butterworth) [48]. The Root mean square (RMS) of the signal was finally computed to measure an amount of activation during one movement and peak activation was computed as the maximum value of the signal for each movement. Possible outliers on peak activation were removed with a threshold at 3 standard deviations. The activity of flexors is computed as the average activity of the brachio-radialis and biceps brachii and the activity of extensors is computed as the average activity of the triceps brachii long head and lateral head.

Robot data: Angular positions of the fourth axis of the robot were recorded by means of internal encoders and angular velocity was estimated by numerical derivation of these positions. Interaction forces were recorded by means of the FT sensor to assess the validity of our compensation. A low-pass filter $(5 \mathrm{~Hz}$ cut-off frequency, Butter-worth) was applied on robot angular velocity. Acceleration of the fourth axis was then obtained by numerical differentiation of the filtered velocity for offline computations. The "real" interaction torque at the participants' elbow $\tau_{i}$ was computed as presented in Equation (18),

$$
\tau_{i}=l F
$$

where $l$ is the distance between the robot elbow and the slider estimated manually at the beginning of the experiment, $F$ is the interaction force measured on the z-axis (i.e. the normal) of the FT sensor. The distance $l$ varies during the experiment but these variations are neglected as they are of small magnitude when compared with the initial distance and have little impact on the weight compensation torque. Theoretical $F$ and $\tau_{i}$ were also computed using the model presented in Equation (12). In all our experiments we fully compensated weight $(\alpha=1)$, therefore the condition $\tau_{i}>0 \mathrm{Nm}$ should be respected.

Statistical analysis: The inter-individuals data were first compared via repeated measures ANOVA with a correction of Greenhouse-Geisser. If statistical differences are observed, data sets are compared using pairwise $t$-test comparisons with a Bonferroni correction as post-hoc tests. All statistical analyses are conducted with the Pingouin package [43]. All significance levels are fixed at $p<0.05$.

\section{Results}

\subsection{Impact of JM consideration and identified human arm parameters}

In this subsection, we compare the identification errors for the models with and without JM and report the identified human arm parameters in each case to validate our approach.

The results of identification errors are given in Figure 6. 


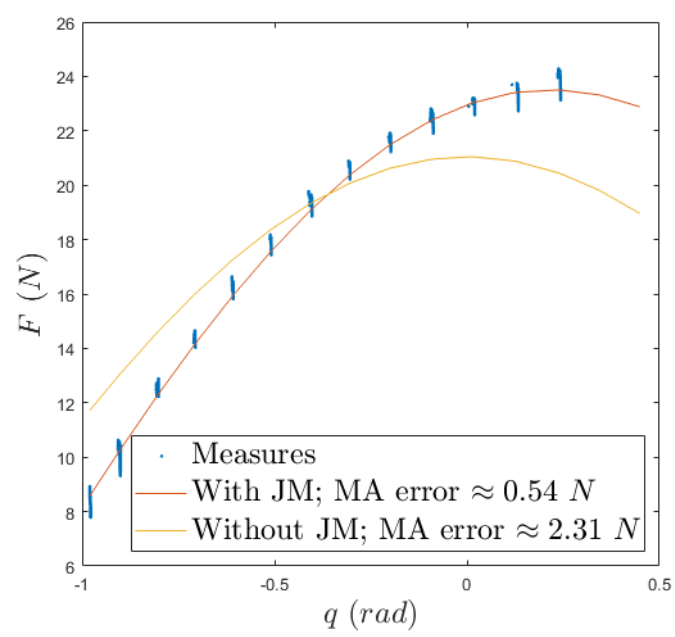

(a)

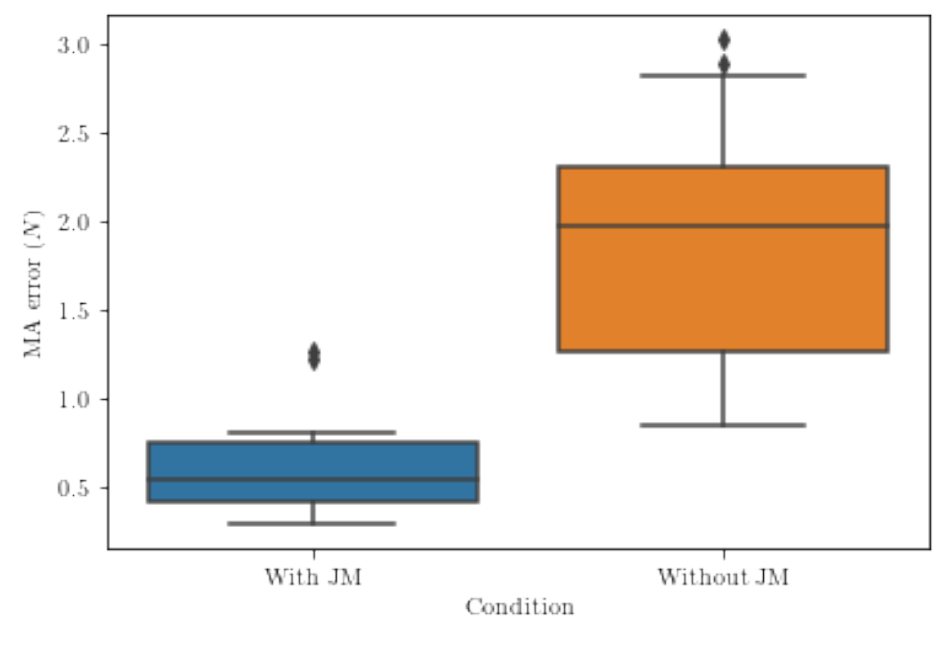

(b)

Figure 6: Effects of JM on model behavior and MAEs. (a) Example of differences in model identification for a representative participant. (b) Boxplots representing MAEs repartition with and without JM.

\begin{tabular}{ccc} 
Method & $\begin{array}{c}\text { Mean } \\
\text { MAE }(N)\end{array}$ & $\begin{array}{c}\text { Standard } \\
\text { deviation }(N)\end{array}$ \\
\hline Without JM, $\phi \triangleq 0$ & 1.9 & 0.68 \\
With JM, $\phi \in \Re$ & 0.62 & 0.28 \\
\hline
\end{tabular}

Table 3: Mean and standard deviation of MAEs for both identification methods.

An example of the differences between the two identified models is given in Figure 6a. This example clearly shows that considering JM corrects a substantial amount of error. The arm parameters identified in this example were $m_{e q, h} \approx 2.45 \mathrm{~kg} ; \phi \approx 0.217 \mathrm{rad}$ with JM and $m_{e q, h} \approx 2.14 \mathrm{~kg}$ without. The identification MAEs across participants are displayed for both approaches in Figure 6b, showing the distribution of identification errors both with and without JM through boxplots. The pairwise $t$-test returned a significant difference between the two sets ( $T$-value: -7.18 , $\left.p=3.91 \times 10^{-7}\right)$. Mean and standard deviation of the MAEs are given in Table 3, showing a net decrease in mean error and in error variability. Indeed, the mean error is approximately divided by a factor 3 and the standard deviation is approximately divided by a factor 2.4 .

The mean identified $m_{e q, h}$ and $\phi_{h}$ and their standard deviations for all participants are given in Table 4 .

\begin{tabular}{ccc} 
Identified parameter & $m_{e q, h}(\mathrm{~kg})$ & $\phi_{h}(\mathrm{rad})$ \\
\hline Mean & 1.93 & 0.21 \\
Standard deviation & 0.26 & 0.07 \\
\hline
\end{tabular}

Table 4: Mean and standard deviation of mass and $\phi_{h}$ values across participants.
These results demonstrate the prominent role of JM in achieving a correct human segment mass identification, which is a necessary prerequisite towards an accurate WS. Furthermore, the estimation of the position of the user in the exoskeleton is a data that could be used in other situations such as tasks based on assistive control laws.

As the WS model has now been defined and validated, the next step is the validation of the WS control law designed in Section 2.3.1. This validation is presented in Section 3.2.

\subsection{Comparison of the performance of control laws}

Comparison of control laws static performances: An important feature of WS control laws is the ability to remain static when the user is relaxed. Therefore the measurement of errors between the identified model and the measured efforts in static conditions is necessary to quantify the overall quality of these control laws. In the present study, these errors were measured on 5-second pointing on each of the three targets $\left(T_{u p}\right.$, $T_{b o t}$ and $\left.T_{m i d}\right)$. The obtained results are given in Figure 7 .

The results depicted in Figure 7 show a net decrease of the torque-level MAEs by using the FFTF and FFCM control laws.The FFCM control law seems to induce a second reduction of the static MAEs overall. It also seems that this control law induces a reduction in MAE variability for $T_{m i d}$ and $T_{u p}$. Both the FFTF and FFCM control laws induce a net decrease in MAE variability when compared with the $\mathrm{BC}$ control law. 


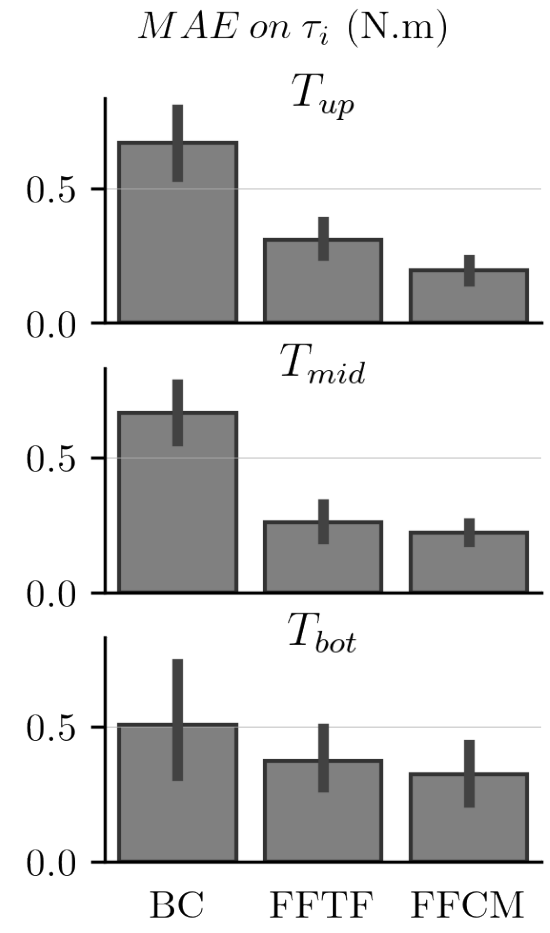

Figure 7: Comparison of the control laws' performances under static conditions.

The repeated measures ANOVA on control laws and targets returns significant differences between conditions $\left(F_{2,36}=36.7, p=0.3 \times 10^{-5}, \eta^{2}=0.67\right)$. No statistical differences are observed between targets. The interaction between targets and control laws is not significant either. Post-hoc treatments show that FFTF and FFCM are significantly better than BC overall ( $p=1.1 \times 10^{-4}$ and $p=0.7 \times 10^{-5}$ respectively $)$. Furthermore, post-hoc treatments show that FFCM offers a significantly better performance than FFTF $\left(p=2.5 \times 10^{-3}\right)$ in terms of MAE on the three targets.

Tracking performance: One objective of the present study was to design an accurate WS control law in terms of weight model tracking. Therefore, the best control law must minimize the differences between the predicted interaction force described in Section 2.1 and the measured interaction force $F$. The mean results in terms of model tracking for each control law are visible in the third row of Figure 9. Movement traces averaged for one participant are reported for upward movements in Figure 9.

The quantification of the performance of each control law in terms of weight model tracking (i.e. MAE repartition during the whole blocks) is presented in Figure 8.

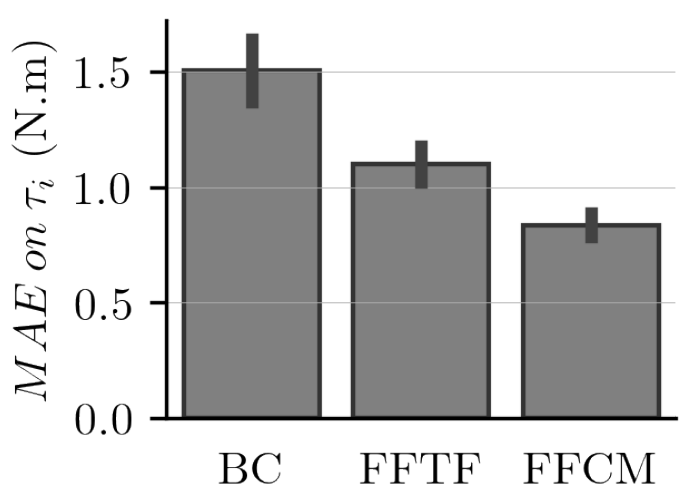

Figure 8: Comparison of the tracking performance each control law in terms of MAE on $\tau_{i}$ for each experimental session.

Overall, the FFCM control law performs better (lower average value and with a smaller standard deviation than other laws). This figure reveals a substantial reduction of the MAEs with the addition of each feedforward term. A decrease of approximately $27 \%$ of the mean MAE when adding the feedforward term based on the error relative to the theoretical force. The addition of the term based on the error relative to the angular position results in a decrease of approximately $24 \%$ of the mean error when comparing FFTF and FFCM and of approximately $44 \%$ when comparing BC and FFCM. The variability of the mean MAE also shows a substantial decrease when adding the feedforward terms. These results are promising considering that the aim of the present study is to build a law suitable for the entire population.

The ANOVA analysis returns a significant difference between the different laws $\left(p=2.49 \times 10^{-11}, F=\right.$ $54.5)$. The paired $t$-tests conducted on these MAEs all reveal significant improvements in terms of tracking performances: $\mathrm{BC}$ vs FFTF returns $p=9.3 \times$ $10^{-5}, T=5.61, \mathrm{BC}$ vs FFCM returns $p=5.54 \times$ $10^{-8}, T=9.88$ and FFTF vs FFCM returns $p=$ $2.85 \times 10^{-4}, T=5.07$.

Eventually, the performance in terms of MAE under dynamic and static conditions is proven to increase with both the FFTF and FFCM control laws, which supports their validity and shows the relevance of their design method a posteriori. Nevertheless, the question of the effects of these control laws on human movement and physiological behavior is still unanswered. This is why these effects are the main focus of Section 3.3.

3.3 Effects on human trajectories and muscle activity Trajectories: The movement profiles obtained with the three WS control laws were all globally similar 
to the transparent condition with bell-shaped velocity profiles (see Figure 9 for details on upward movements). Furthermore, Figure 9 does not seem to suggest that there is more overshoot with WS control laws than in transparent mode. This is confirmed by a repeated measures ANOVA on the first sub-movement amplitude that showed no significant difference between the conditions $\left(F_{3,54}=0.446, p=0.721\right.$, $\left.\eta^{2}=0.0242\right)$ This suggests that participants successfully adapted their motor planning and correction to WS control laws.

Regarding the basic kinematic features of the movements, we report below the movement duration, peak velocity and peak acceleration in each condition. These averaged values are reported in Table 5. These values were computed on all the movements (pooling both upward and downward movements).

\begin{tabular}{cccc} 
& PV & PA & D \\
& $(\mathrm{rad} / \mathrm{s})$ & $\left(\mathrm{rad} / \mathrm{s}^{2}\right)$ & $(\mathrm{s})$ \\
\hline TR & $29.1 \pm 6.8$ & $157.4 \pm 56.2$ & $0.64 \pm 0.14$ \\
BC & $28.8 \pm 6.5$ & $155.8 \pm 52.3$ & $0.64 \pm 0.17$ \\
FFTF & $30.9 \pm 6.9$ & $169.7 \pm 60.8$ & $0.61 \pm 0.15$ \\
FFCM & $29.8 \pm 6.4$ & $165.3 \pm 56.5$ & $0.61 \pm 0.12$ \\
\hline
\end{tabular}

Table 5: Means and standard deviations of standard descriptors of human movement. PV stands for Peak Velocity, PA stands for Peak Acceleration and D stands for Duration.

Mean values do not show any clear difference between the control laws on these parameters. Peak velocities, peak accelerations and movement duration were similar for all control laws. This was confirmed by repeated measures ANOVA which showed no significant difference between all the control laws for these parameters.

Muscle activity: As for the analysis of the performance of control laws in Section 3.2, data of EMG sensors were analyzed under two situations: during static positions maintained at the end of the blocks and during dynamic movements. For the latter situation, we focused on the muscular activity during the acceleration phase, which reflects the effort provided by the user to launch the movement and is related to the obtained peak acceleration.

The mean results in terms of RMS of EMG signals at the three targets during the static part of the blocks are illustrated by Figure 10 .

These results suggest that there is a significant reduction in flexor muscle activity while maintaining static positions. This is confirmed by the repeated measures ANOVA conducted on these data that reports significant differences between conditions $\left(F_{3,54}=553.14, p=2.82 \times 10^{-28}, \eta^{2}=0.97\right)$. Post-hoc analyses show that the three WS laws (BC, FFTF and FFCM) induce significantly reduced flexors activity by the participant when compared to the $\mathrm{TR}$ condition $\left(p=2.2 \times 10^{-5}, p=2.1 \times 10^{-5}\right.$ and $p=9.5 \times 10^{-5}$ respectively). No statistical differences are observed for extensors activity during post-hoc treatments even though the ANOVA reported a slightly significant main effect $\left(F_{3,54}=3.73, p=0.046, \eta^{2}=0.17\right.$. This result suggests that differences might exist. Nevertheless, these differences might be due to intra-individual differences during the experimentation as the "transparent" block was always the first block. As expected, WS reduces flexors activity while maintaining static positions across the whole movement range and mostly leaves extensors activity unchanged (in agreement with the fact that these muscles are not critical to counteract gravitational loads in this posture).

The second step of the analysis was to quantify the dynamic effects of the three WS laws in the sagittal plane. Therefore, the normalized peak of muscular activity of the initial burst of the agonist muscle is a relevant index as it is linked to the effort planned by the participants. The results obtained for upward and downward movements are illustrated in Figure 11.

These mean results demonstrate a decrease in flexors activity to initiate upward movements with the WS control laws, which is coherent with the expected impact of WS control laws. The results also demonstrate an increase in extensor activity to initiate downward movements (which is also coherent with the theoretical effect of WS which prevents participants from taking advantage of weight to move downward). These findings are confirmed by the results of a repeated measures ANOVA that shows a significant difference in flexor activity between conditions ( $F_{3}=39.82$, $\left.p=3.52 \times 10^{-8}, \eta^{2}=0.69\right)$. Post-hoc analyses report significant differences between the TR and WS conditions for flexors during upward movements $(p=$ $1.01 \times 10^{-7}, p=4.49 \times 10^{-5}$ and $p=4.99 \times 10^{-8}$ for $\mathrm{BC}, \mathrm{FFTF}$ and FFCM respectively). Another repeated measures ANOVA confirms a significant difference in extensors activity between conditions $\left(F_{3}=41.52\right.$, $\left.p=4.73 \times 10^{-14}, \eta^{2}=0.70\right)$. Post-hoc analyses report significant differences between the TR and WS conditions for extensors during downwards movements $\left(p=5.44 \times 10^{-7}, p=3.25 \times 10^{-9}\right.$ and $p=1.46 \times 10^{-5}$ for BC, FFTF and FFCM respectively). No statistical differences are observed between the WS control laws in terms of peak activity for both flexors during upward movements, and extensors during downward movements. As expected WS reduces significantly the activity of flexors during upward movements but increases the activity of extensors activity during downward movements. 


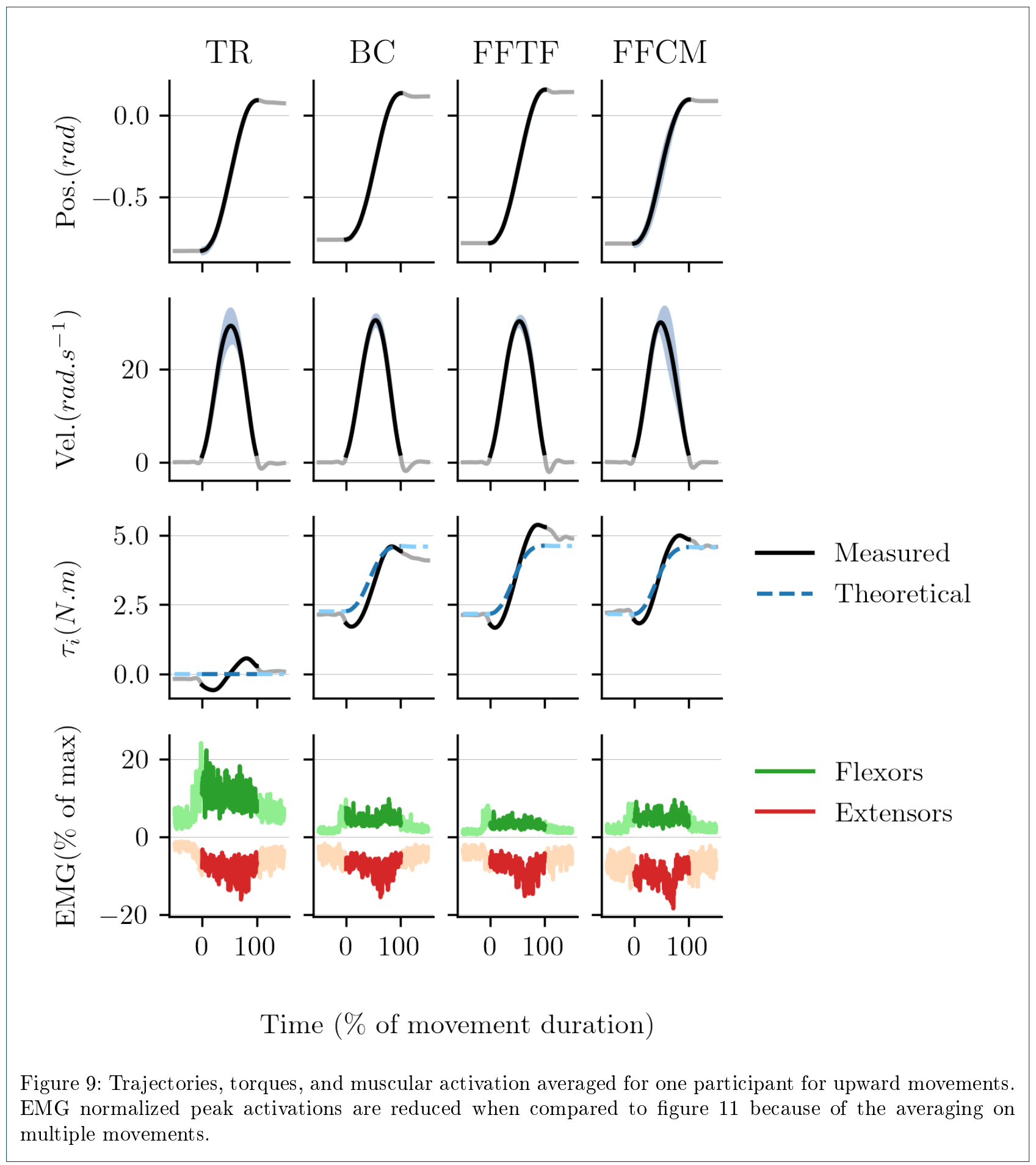

\section{Discussion}

Weight model: The present study introduced a new weight model for WS considering JM between the participant and the exoskeleton. These misalignments have proven to be an important factor to correctly describe the weight of a human segment from the robot's viewpoint. Even though certain exoskeletons can be adjusted to the user to minimize JM [36, 49], they cannot be completely canceled [11] and, therefore, should not be ignored.

Control laws: The present study tested different control strategies to implement an accurate active WS while moving into the sagittal plane where gravita- 


\section{$E M G_{R M S}$ (\% of max) \\ Flexors \\ Extensors}
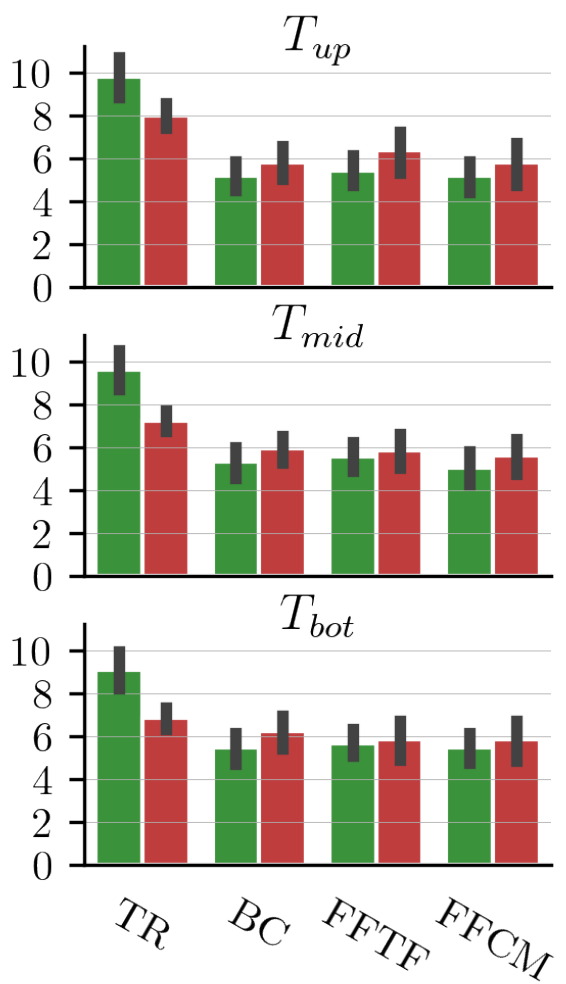

Figure 10: RMS of EMG signals during static position maintenance at the three targets.

tional torques vary. Our results showed the effectiveness of WS both in static and dynamic situations, although we noticed opposite effects on flexors and extensors at movement initiation, as expected [50]. Other studies have focused on the influence of WS on EMG activity and showed an increase in the number of muscular synergies by looking at ensemble EMG patterns $[37,38,39]$. Here we obtained clear improvements in the quality of WS in terms of tracking performance for laws including a feedforward term, although the impact on human muscle activity was only visible in statics in our data. Other studies had already described a weight compensation strategy based on a feedforward model-based control $[8,35]$. Nevertheless, the performance of this control was not quantified in those works, and the present study suggests that classical approaches might not be sufficient to achieve an accurate WS. As a consequence, feedforward terms learned

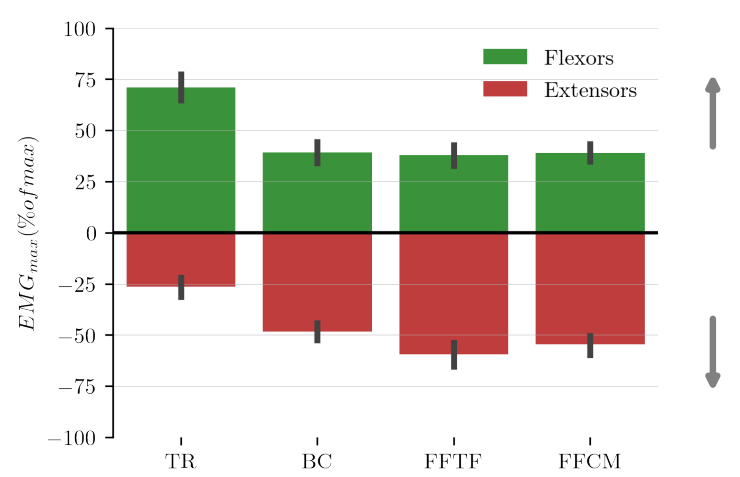

Figure 11: Mean normalized peak of muscular activity of the agonist muscles estimated during the movement acceleration phase. Flexors are agonist for upward movements (depicted with positive values) and extensors are agonist for downward movements (depicted with negative values).

from error measurements during experiments can improve the accuracy of the impedance controller. These feedforward terms are currently limited to linear models as they are simple to implement and to track for the controller.

The main conclusion that can be drawn is that the use of simple feedforward models based on error measurement is a viable solution for the design of effective WS control laws. Indeed, it has been shown that, even for control laws designed from population-based data (and not personalized), the tracking errors can be significantly reduced compared to classic PI control methods. Furthermore, this design method can be individualised easily if needed, which should lead to even better performance. As it can be seen in Figures $3 \mathrm{~b}$ and 9, the defined linear models cannot capture, and therefore compensate, errors due to inertial effects and time delay in the feedback control law. The compensation of these errors could provide an important improvement towards more homogeneous active WS.

Effects on human movement: Previous studies provided evidence for an increase in work-space size and overall motor performance in stroke patients with WS $[15,51,8]$ but also for comparable movement performances in terms of velocity and trajectory [38, 39]. In the present study, basic kinematic parameters were also comparable between the TR condition and all the WS control laws, thereby supporting a relative stability of the overall motion kinematics. Therefore, although WS was quantitatively improved using feedforward terms, its impact on human movement data was not obvious. More accurate analyses focusing on 
the adaptation process or on finer movement parameters could allow to find more subtle effects. For instance, the adaptation of specific movement characteristics such as velocity profile asymmetries, which are well known to be influenced by the ambient gravity field in the motor control literature [13, 52, 53], could vary for different control laws. Also, more advanced analyses of ensemble muscle patterns could be applied to detect more complex changes in muscle activities [54].

The findings of the present study in terms of EMG adaptation are coherent with previous studies that described a decrease in elbow flexors activity when wearing an exoskeleton in WS mode or using a passive device to compensate for weight $[37,38,39,40]$. The present study also provides data on the increase in elbow extensor activity during movements in a vertical plane when using WS. Future control laws could implement a WS control that activates and deactivates so that patients could exploit gravity to start a downward movement or to brake an upward movement [50]. The present results also suggest that, reducing tracking errors had no significant impact on static and dynamic EMG signals even though variations are observed on average. This is probably due to the variability and noise of EMG sensors that do not allow to observe any clear difference with light interaction force differences. Indeed, if interaction force measures are different (which is the case) but kinematic measures are equivalent (which is also the case), then the differences can only be compensated by muscles activation.

\section{Conclusion}

In the present study, the importance of JM to accurately compensate for weight was demonstrated and a general model with an associated identification procedure was given. Then, three WS control laws, designed on the basis of experiments, were introduced and tested. The adopted methodology led to significant improvements in model tracking performances and to a control law suitable for the whole tested population. This methodology is also suitable to design a fully personalized WS control law for rehabilitation applications. The effects on human movements are comparable to those obtained in previous studies. Nevertheless, the measurements of muscular activities suggest that a partial WS, that activates only when it helps to reduce muscular effort, could be more suitable for rehabilitation applications than a complete WS control mode.

\section{Appendix}

Definition of rotation matrices: The rotation between the robot link and the world frame expressed in the world frame is presented in Equation (19),

$$
R_{r 0}\left(\mathbf{z}_{\mathbf{0}}, \phi_{r}\right)=\left(\begin{array}{ccc}
\cos \left(\phi_{r}\right) & -\sin \left(\phi_{r}\right) & 0 \\
\sin \left(\phi_{r}\right) & \cos \left(\phi_{r}\right) & 0 \\
0 & 0 & 1
\end{array}\right)
$$

The rotation between the robot link and the projected human segment expressed in the world frame is presented in Equation (20),

$$
R_{h r, 1}\left(\mathbf{z}_{\mathbf{0}}, \phi_{h}\left(\phi_{r}\right)\right)=\left(\begin{array}{ccc}
\cos \left(\phi_{h}\left(\phi_{r}\right)\right) & -\sin \left(\phi_{h}\left(\phi_{r}\right)\right) & 0 \\
\sin \left(\phi_{h}\left(\phi_{r}\right)\right) & \cos \left(\phi_{h}\left(\phi_{r}\right)\right) & 0 \\
0 & 0 & 1
\end{array}\right)
$$

The rotation between the projected human segment and the human segment expressed in the projected human segment frame is presented in Equation (20),

$$
R_{h r, 2}\left(\mathbf{y}_{\mathbf{h}, \mathbf{p}}, \psi_{h}\left(\phi_{r}\right)\right)=\left(\begin{array}{ccc}
\cos \left(\psi_{h}\left(\phi_{r}\right)\right) & 0 & \sin \left(\psi_{h}\left(\phi_{r}\right)\right) \\
0 & 1 & 0 \\
-\sin \left(\psi_{h}\left(\phi_{r}\right)\right) & 0 & \cos \left(\psi_{h}\left(\phi_{r}\right)\right)
\end{array}\right)
$$

The rotation between the world frame and the projected human segment expressed in the world frame is presented in Equation (20),

$$
R_{h 0}\left(\mathbf{z}_{\mathbf{0}}, \phi_{r}+\phi_{h}\left(\phi_{r}\right)\right)=\left(\begin{array}{ccc}
\cos \left(\phi_{r}+\phi_{h}\left(\phi_{r}\right)\right) & -\sin \left(\phi_{r}+\phi_{h}\left(\phi_{r}\right)\right) & 0 \\
\sin \left(\phi_{r}+\phi_{h}\left(\phi_{r}\right)\right) & \cos \left(\phi_{r}+\phi_{h}\left(\phi_{r}\right)\right) & 0 \\
0 & 0 & 1
\end{array}\right)
$$


Ethics approval and consent to participate

All studies were approved by the responsible institutions (Université Paris-Saclay, 2017-34)

\section{Consent for publication}

Written informed consent was obtained from all human subjects for the publication of this report and any accompanying images.

\section{Availability of data and materials}

The dataset used and/or analysed during the current study are available from the corresponding author upon reasonable request.

\section{Competing interests}

The authors declare that they have no competing interests.

\section{Funding}

This work is supported by the "IDI 2017" project funded by the IDEX Paris-Saclay, ANR-11-IDEX-0003-02. This work is supported by the French National Agency for Research (grant ANR-19-CE33-0009).

\section{Authors' contributions}

DV (dorian.verdel Ouniversite-paris-saclay.fr) performed the third experimental session, designed the control laws and redacted the core of the manuscript. SB (simon.bastide@universite-paris-saclay.fr) performed the first two experimental sessions and treated the data. NV (nicolas.vignais@universite-paris-saclay.fr), OB

(olivier.bruneau@ens-paris-saclay.fr) and BB (bastien.berret@universite-paris-saclay.fr) oriented the research and provided remarks on the manuscript redaction. All authors read and approved the final manuscript.

\section{Acknowledgements}

No acknowledgements.

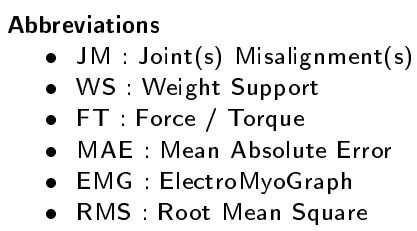

\section{Author details}

${ }^{1}$ CIAMS, Université Paris-Saclay, Orsay, France. ${ }^{2} \mathrm{CIAMS}$, Université d'Orléans, Orléans, France. ${ }^{3}$ LURPA, ENS Paris-Saclay, Gif-sur-Yvette, France. ${ }^{4}$, Institut Universitaire de France, Paris, France.

\section{References}

1. Frisoli, A., Borelli, L., Montagner, A., Marcheschi, S., Procopio, C., Salsedo, F., Bergamasco, M., Carboncini, M.C., Tolaini, M., Rossi, B.: Arm rehabilitation with a robotic exoskeleleton in Virtual Reality. In: IEEE 10th International Conference on Rehabilitation Robotics ICORR, 2007., pp. 631-642 (2007). doi:10.1109/ICORR.2007.4428491

2. Frisoli, A., Procopio, C., Chisari, C., Creatini, I., Bonfiglio, L., Bergamasco, M., Rossi, B., Carboncini, M.C.: Positive effects of robotic exoskeleton training of upper limb reaching movements after stroke. Journal of neuroengineering and rehabilitation 9(1), 36 (2012). doi:10.1186/1743-0003-9-36

3. Huang, V.S., Krakauer, J.W.: Robotic neurorehabilitation: a computational motor learning perspective. Journal of NeuroEngineering and Rehabilitation 6(5), 1-13 (2009)

4. Benabid, Y., Chettibi, T., Aoussat, A., Benfriha, K.: Design and implementation of orthosis to improve gait of patients with hemiplegia. Computer Methods in Biomechanics and Biomedical Engineering 15(sup1), 345-347 (2012). doi:10.1080/10255842.2012.713633

5. Mooney, L.M., Rouse, E.J., Herr, H.M.: Autonomous exoskeleton reduces metabolic cost of human walking during load carriage. Journal of NeuroEngineering and Rehabilitation 11(1), 80 (2014). doi:10.1186/1743-0003-11-80

6. Huo, W., Arnez-Paniagua, V., Ding, G., Amirat, Y., Samer, M.: Adaptive proxy-based controller of an active ankle foot orthosis to assist lower limb movements of paretic patients. Robotica 37(12), 2147-2164 (2019). doi:10.1017/S0263574719000250
7. Ajoudani, A., Zanchettin, A.M., Ivaldi, S., Albu-Schäffer, A., Kosuge, K., Khatib, O.: Progress and prospects of the human-robot collaboration. Autonomous Robots, 957-975 (2018). doi:10.1007/s10514-017-9677-2

8. Just, F., Özhan Özen, Tortora, S., Klamroth-Marganska, V., Riener, R., Rauter, G.: Human arm weight compensation in rehabilitation robotics: efficacy of three distinct methods. Journal of NeuroEngineering and Rehabilitation 17(1) (2020). doi:10.1186/s12984-020-0644-3

9. Proietti, T., Crocher, V., Roby-Brami, A., Jarrasse, N.: Upper-Limb Robotic Exoskeletons for Neurorehabilitation: A Review on Control Strategies. IEEE Reviews in Biomedical Engineering 9, 4-14 (2016). doi:10.1109/RBME.2016.2552201

10. Jarrasse, N., Paik, J., Pasqui, V., Morel, G.: How can human motion prediction increase transparency? IEEE International Conference on Robotics and Automation, 2134-2139 (2008). doi:10.1109/ROBOT.2008.4543522

11. Jarrasse, N., Morel, G.: Connecting a Human Limb to an Exoskeleton. IEEE Transactions on Robotics 28(3), 697-709 (2012). doi:10.1109/TRO.2011.2178151

12. Pirondini, E., Coscia, M., Marcheschi, S., Roas, G., Salsedo, F., Frisoli, A., Bergamasco, M., Micera, S.: Evaluation of the effects of the Arm Light Exoskeleton on movement execution and muscle activities: a pilot study on healthy subjects. Journal of NeuroEngineering and Rehabilitation (2016). doi:10.1186/s12984-016-0117-x

13. Bastide, S., Vignais, N., Geffard, F., Berret, B.: Interacting with a "transparent" upper-limb exoskeleton: a human motor control approach. IEEE/RSJ International Conference on Intelligent Robots and Systems (IROS), 4661-4666 (2018). doi:10.1109/IROS.2018.8593991

14. Verdel, D., Bastide, S., Vignais, N., Bruneau, O., Berret, B.: An identification-based method improving the transparency of a robotic upper-limb exoskeleton. Robotica (2021). doi:10.1017/S0263574720001459

15. Prange, G.B., Jannink, M.J.A., Groothuis-Oudshoorn, C.G.M., Hermens, H.J., IJzerman, M.J.: Systematic review of the effect of robot-aided therapy on recovery of the hemiparetic arm after stroke. The Journal of Rehabilitation Research and Development 43(2), 171 (2006). doi:10.1682/jrrd.2005.04.0076

16. Theurel, J., Desbrosses, K., Roux, T., Savescu, A.: Physiological consequences of using an upper limb exoskeleton during manual handling tasks. Applied Ergonomics 67, 211-217 (2018). doi:10.1016/j.apergo.2017.10.008

17. Treussart, B., Geffard, F., Vignais, N., Marin, F.: Controlling an upper-limb exoskeleton by EMG signal while carrying unknown load. In : 2020 IEEE International Conference on Robotics and Automation (ICRA), pp. 9107-9113 (2020). doi:10.1109/icra40945.2020.9197087

18. Tyryshkin, K., Coderre, A.M., Glasgow, J.I., Herter, T.M., Bagg, S.D., Dukelow, S.P., Scott, S.H.: A robotic object hitting task to quantify sensorimotor impairments in participants with stroke. Journal of NeuroEngineering and Rehabilitation 11(1), 47 (2014). doi:10.1186/1743-0003-11-47

19. Otaka, E., Otaka, Y., Kasuga, S., Nishimoto, A., Yamazaki, K., Kawakami, M., Ushiba, J., Liu, M.: Clinical usefulness and validity of robotic measures of reaching movement in hemiparetic stroke patients. Journal of NeuroEngineering and Rehabilitation 12(1) (2015). doi:10.1186/s12984-015-0059-8

20. Mochizuki, G., Centen, A., Resnick, M., Lowrey, C., Dukelow, S.P., Scott, S.H.: Movement kinematics and proprioception in post-stroke spasticity: assessment using the kinarm robotic exoskeleton. Journal of NeuroEngineering and Rehabilitation 16(1) (2019). doi:10.1186/s12984-019-0618-5

21. Carson, R.G., Oytam, Y., Riek, S.: Artificial gravity reveals that economy of action determines the stability of sensorimotor coordination. PLoS ONE 4(4), 5248 (2009). doi:10.1371/journal.pone.0005248

22. Oytam, Y., Lloyd, D., Reid, C.S., de Rugy, A., Carson, R.G.: A robotic apparatus that dictates torque fields around joints without affecting inherent joint dynamics. Human Movement Science 29(5), 
701-712 (2010). doi:10.1016/j.humov.2010.06.004

23. Hatze, H.: Parameter identification for human body segment models. Theoretical Issues in Ergonomics Science 6(3-4), 331-334 (2005). doi:10.1080/14639220512331329509

24. Chandler, R.F., Clauser C., E., McConviRe J., T., Reynolds H., M., Young, J.W.: Investigation of inertial properties of the human body. (AMRL TR 74-137). Wright-PattersonAir Force Base, Ohio (NTIS No. ADA016 485) (1975)

25. de Leva, P.: Adjustments to zatsiorsky-seluyanov's segment inertia parameters. Journal of Biomechanics 29(9), 1223-1230 (1996). doi:10.1016/0021-9290(95)00178-6

26. de Leva, P.: Joint center longitudinal positions computed from a selected subset of chandler's data. Journal of Biomechanics 29(9), 1231-1233 (1996). doi:10.1016/0021-9290(96)00021-8

27. Winter, D.A.: Biomechanics and Motor Control of Human Movement, 2nd edn. John Wiley and Sons, vol. 1. New York: John Wiley and Sons, New York (1990)

28. Todorov, E.: Probabilistic inference of multijoint movements, skeletal parameters and marker attachments from diverse motion capture data. IEEE Transactions on Biomedical Engineering 54(11), 1927-1939 (2007). doi:10.1109/tbme.2007.903521

29. Venture, G., Ayusawa, K., Nakamura, Y.: Motion capture based identification of the human body inertial parameters. In: 30th Annual International Conference of the IEEE Engineering in Medicine and Biology Society (2008). doi:10.1109/iembs.2008.4650231

30. Jovic, J., Escande, A., Ayusawa, K., Yoshida, E., Kheddar, A., Venture, G.: Humanoid and human inertia parameter identification using hierarchical optimization. IEEE Transactions on Robotics 32(3), 726-735 (2016). doi:10.1109/tro.2016.2558190

31. Venture, G., Yamane, K., Nakamura, Y., Hirashima, M.: Estimating viscoelastic properties of human limb joints based on motion capture and robotic identification technologies. In: 2007 IEEE/RS J International Conference on Intelligent Robots and Systems (2007) doi:10.1109/iros.2007.4398958

32. Venture, G., Ayusawa, K., Nakamura, Y.: Real-time identification and visualization of human segment parameters. In: 2009 Annual International Conference of the IEEE Engineering in Medicine and Biology Society (2009). doi:10.1109/iembs.2009.5333620

33. Ayusawa, K., Venture, G., Nakamura, Y.: Real-time implementation of physically consistent identification of human body segments. In: 2011 IEEE International Conference on Robotics and Automation (2011). doi:10.1109/icra.2011.5979903

34. Bonnet, V., Fraisse, P., Crosnier, A., Gautier, M., Gonzalez, A., Venture, G.: Optimal exciting dance for identifying inertial parameters of an anthropomorphic structure. IEEE Transactions on Robotics 32(4), 823-836 (2016). doi:10.1109/tro.2016.2583062

35. Just, F., Ozen, O., Tortora, S., Riener, R., Rauter, G.: Feedforward model based arm weight compensation with the rehabilitation robot ARMin. In : 2017 International Conference on Rehabilitation Robotics (ICORR) (2017). doi:10.1109/icorr.2017.8009224

36. Hwang, B., Jeon, D.: A method to accurately estimate the muscular torques of human wearing exoskeletons by torque sensors. Sensors 15(4), 8337-8357 (2015). doi:10.3390/s150408337

37. F. Beer, R., D. Ellis, M., Bradley, G.H., Julius, P.A.D.: Impact of gravity loading on post-stroke reaching and its relationship to weakness. Muscle and Nerve 36(2), 242-250 (2007). doi:10.1002/mus.20817

38. Prange, G.B., Jannink, M.J.A., Stienen, A.H.A., van der Kooij, H., IJzerman, M.J., Hermens, H.J.: Influence of gravity compensation on muscle activation patterns during different temporal phases of arm movements of stroke patients. Neurorehabilitation and Neural Repair 23(5), 478-485 (2009). doi:10.1177/1545968308328720

39. Prange, G.B., Kallenberg, L.A.C., Jannink, M.J.A., Stienen, A.H.A., van der Kooij, H., IJzerman, M.J., Hermens, H.J.: Influence of gravity compensation on muscle activity uring reach and retrieval in healthy elderly. Journal of Electromyography and Kinesiology 19(2), 40-49 (2009). doi:10.1016/j.jelekin.2007.08.001

40. Coscia, M., Cheung, V.C., Tropea, P., Koenig, A., Monaco, V., Bennis, C., Micera, S., Bonato, P.: The effect of arm weight support on upper limb muscle synergies during reaching movements. Journal of NeuroEngineering and Rehabilitation 11(22), 1-15 (2014). doi:10.1186/1743-0003-11-22

41. Runnalls, K.D., Ortega-Auriol, P., J C McMorland, A., Anson, G., D Byblow, W.: Effects of arm weight support on neuromuscular activation during reaching in chronic stroke patients. Experimental Brain Research 237(12), 3391-3408 (2019). doi:10.1007/s00221-019-05687-9

42. World Medical Association.: World Medical Association Declaration of Helsinki. Ethical principles for medical research involving human subjects. Bulletin of the World Health Organization 79(4), 373-374 (2001)

43. Vallat, R.: Pingouin: statistics in python. Journal of Open Source Software 3(31), 1026 (2018). doi:10.21105/joss.01026

44. Verdel, D., Bastide, S., Bruneau, O., Berret, B., Vignais, N.: Improving and quantifying the transparency of an upper-limb robotic exoskeleton with a force sensor and electromyographic measures. Computer Methods in Biomechanics and Biomedical Engineering (2021 (In press))

45. Garrec, P., Friconneau, J.P., Méasson, Y., Perrot, Y.: ABLE, an Innovative Transparent Exoskeleton for the Upper-Limb. IEEE/RSJ International Conference on Intelligent Robots and Systems (IROS), 1483-1488 (2008). doi:10.1109/IROS.2008.4651012

46. Garrec, P.: Screw and Cable Acutators (SCS) and Their Applications to Force Feedback Teleoperation, Exoskeleton and Anthropomorphic Robotics. Robotics 2010 Current and Future Challenges, 167-191 (2010). doi:10.5772/7327

47. Hermens, H.J., BV, R.R.a.D. (eds.): European Recommendations for Surface ElectroMyoGraphy: Results of the SENIAM Project. SENIAM, vol. 8. Roessingh Research and Development, Enschede (1999)

48. Potvin, J.R., Brown, S.H.M.: Less is more: high pass filtering, to remove up to $99 \%$ of the surface EMG signal power, improves EMG-based biceps brachii muscle force estimates. Journal of Electromyography and Kinesiology 14(3), 389-399 (2004). doi: $10.1016 / j$.jelekin.2003.10.005

49. Kong, K., Jeon, D.: Design and control of an exoskeleton for the elderly and patients. IEEE/ASME Transactions on Mechatronics 11(4), 428-432 (2006), doi:10.1109/tmech.2006.878550

50. Gaveau, J., Grospretre, S., Angelaki, D., Papaxanthis, C.: A cross-species neural integration of gravity for motor optimisation. Science Advances 7(15) (2019). doi:10.1126/sciadv.abf7800

51. Ellis, M.D., Sukal-Moulton, T.M., Dewald, J.: Impairment-based 3-d robotic intervention improves upper extremity work area in chronic stroke: targeting abnormal joint torque coupling with progressive shoulder abduction loading. IEEE Transactions on Robotics 25(3), 549-555 (2009). doi:10.1109/tro.2009.2017111

52. Gentili, R., Cahouet, V., Papaxanthis, C.: Motor planning of arm movements is direction-dependent in the gravity field. Neuroscience (145), 20-32 (2007). doi:10.1016/j.neuroscience.2006.11.035

53. Gaveau, J., Berret, B., Angelaki, D.E., Papaxanthis, C.: Direction-dependent arm kinematics reveal optimal integration of gravity cues. eLife 5, 1-17 (2016). doi:10.7554/eLife.16394

54. Chiovetto, E., Berret, B., Delis, I., Panzeri, S., Pozzo, T.: Investigating reduction of dimensionality during single-joint elbow movements: a case study on muscle synergies. Frontiers in Computational Neuroscience 7 (2013). doi:10.3389/fncom.2013.00011 